\title{
Ground Moving Target Imaging using Bi-static Synthetic Aperture Radar
}

\author{
Kaan Duman \\ Department of Electrical, \\ Computer and Systems Engineering, \\ Rensselaer Polytechnic Institute, \\ 110 8th Street, Troy, NY 12180 USA \\ Email: dumank@rpi.edu
}

\author{
Birsen Yazıcı \\ Department of Electrical, \\ Computer and Systems Engineering, \\ Rensselaer Polytechnic Institute, \\ 110 8th Street, Troy, NY 12180 USA \\ Email: yazici@ecse.rpi.edu
}

\begin{abstract}
In this paper, we present a novel method for imaging of moving targets using bi-static synthetic aperture radar configurations. We present a forward model that maps the twodimensional reflectivity and velocity of targets to the measured scattered field data. We then introduce a filtered-backprojection type method to reconstruct the reflectivity and use Renyi entropy to determine the two-dimensional velocity of targets. The filter is determined so that the reflectivity images are reconstructed at the correct location, orientation and strength whenever the velocity field is determined correctly. We present numerical simulations to verify our theory.
\end{abstract}

\section{INTRODUCTION}

A Bi-static Synthetic Aperture Radar (Bi-SAR) system consists of a moving transmitter and a receiver that are sufficiently far apart. Such a configuration has a number of advantages over a mono-static synthetic aperture radar system where the transmitter and receiver are collocated. In a bi-static configuration, the transmitter with its heavy power supply can be deployed on a separate platform than the inexpensive and expendable receivers, providing system robustness and scalability [1]. Additionally, the electronic counter measures that are designed to thwart mono-static configurations are less effective against bi-static configurations [2], [3].

In this paper we present a novel ground moving target imaging (GMTI) method using Bi-SAR configurations. Fig. 1 depicts a typical Bi-SAR imaging geometry. The objective of the GMTI is to determine the reflectivity (position) as well as the velocity of moving targets [4]. Many methods have been proposed on SAR GMTI for systems using multiple antennas. (See, for example, [5], [6] and [7].) In [5], weighted signals at the receivers are correlated with each other and a likelihood ratio test is performed on the resulting ambiguity function for a hypothetical range and velocity. [6] uses space-time adaptive processing (STAP) for moving target detection. As an alternative to the computationally expensive STAP technique, [7] uses multiple receivers to form velocity versus cross-range images for a set of ranges in an iterative adaptive approach. [8] uses both mono-static and bi-static antennas and extracts motion parameters by using interferometric techniques.

We assume a single transmitter and a single receiver system and reconstruct a set of reflectivity images for a range of hypothesized velocities. We measure the degree to which the reconstructed reflectivity images are focused using the entropy measure and estimate two-dimensional velocity of targets. Our approach is conceptually similar to [4] and [9] where we reconstruct a four dimensional image, two of which corresponds to ground target velocity. Our method has the following advantages: $i$ ) It estimates the velocity of targets irrespective of their direction and speed. ii) It applies to arbitrary imaging geometries including arbitrary antenna trajectories and nonflat topography. iii) It has the advantage of computational efficiency when fast-backprojection algorithms are employed [10].

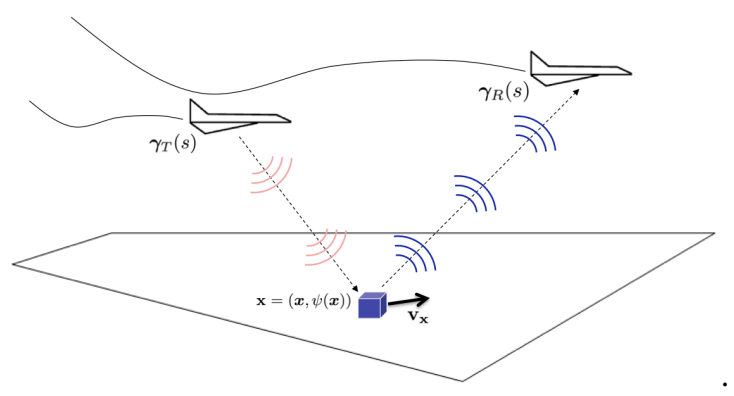

Fig. 1. Acquisition geometry of Bi-SAR GMTI

We first present a forward model to map the reflectivity and velocity of a moving scene to the scattered field data. Next, we provide a filtered-back projection (FBP) type inversion method to reconstruct reflectivity of the scene using different hypothesized velocities. We design the filter to provide wellfocused images of moving targets whenever the hypothesized velocity field is equal to the correct velocity field. We use Renyi entropy as a figure of merit to measure the degree to which reflectivity images are focused and to estimate their correct velocity.

The paper is organized as follows: in section 2 we present our forward model for moving targets in a Bi-SAR system. Section 3 describes the image formation method followed by the velocity determination method. In Section 4, we present numerical simulations. Section 5 concludes our paper. 


\section{FORWARD MODEL}

We let $\gamma_{T}(s) \in \mathbb{R}^{3}$ and $\gamma_{R}(s) \in \mathbb{R}^{3}, s \in\left[s_{0}, s_{1}\right] \subseteq \mathbb{R}$ denote the transmitter and receiver trajectories, respectively where $s$ denotes the slow-time parameter. Let $\mathbf{x}=(\boldsymbol{x}, \psi(\boldsymbol{x})) \in$ $\mathbb{R}^{3}$ denote a location on the ground, where $\boldsymbol{x} \in \mathbb{R}^{2}$ and $\psi: \mathbb{R}^{2} \rightarrow \mathbb{R}$ is a known, smooth function of the ground topography. As usual, we assume that the scattering takes place in a thin region near the surface. Let $\boldsymbol{v}_{\boldsymbol{x}} \in \mathbb{R}^{2}$ denote the 2-D velocity of the target located at $\boldsymbol{x}, D \psi(\boldsymbol{x})$ denote the gradient of $\psi(\boldsymbol{x})$ and $\mathbf{v}_{\mathbf{x}}=\left[\boldsymbol{v}_{\boldsymbol{x}}, D \psi(\boldsymbol{x}) \cdot \boldsymbol{v}_{\mathbf{x}}\right]$ be the 3-D velocity of the ground moving target.

We assume that the antennas are in the far-field of the scene and model the received signal $d(s, t)$ as follows:

$$
\begin{aligned}
& d(s, t)=\mathcal{F}[q](s, t) \\
& \quad:=\int e^{-\mathrm{i} \phi_{T R}(\omega, s, t, \boldsymbol{x}, \boldsymbol{v})} A_{T R}(\omega, s, \boldsymbol{x}, \boldsymbol{v}) q(\boldsymbol{x}, \boldsymbol{v}) d \boldsymbol{x} d \boldsymbol{v} d \omega,
\end{aligned}
$$

where we refer to $q(\boldsymbol{x}, \boldsymbol{v})$ as the phase-space reflectivity function of a moving scene, $t$ denotes the fast-time variable, $c_{0}$ is the speed of light in free space, $\omega$ denotes the temporal frequency and

$$
\phi_{T R}(\omega, s, t, \boldsymbol{x}, \boldsymbol{v})=\omega\left(t-\left[R_{T R}(s, \boldsymbol{x})+B_{T R}(s, \boldsymbol{x}, \boldsymbol{v})\right] / c_{0}\right),
$$

$$
\begin{aligned}
R_{T R}(s, \boldsymbol{x}) & =\left|\mathbf{x}-\gamma_{R}(s)\right|+\left|\mathbf{x}-\gamma_{T}(s)\right|, \\
B_{T R}(s, \boldsymbol{x}, \boldsymbol{v}) & =\left[\overline{\left(\mathbf{x}-\gamma_{R}(s)\right)}+\overline{\left(\mathbf{x}-\gamma_{T}(s)\right)}\right] \cdot \mathbf{v} s .
\end{aligned}
$$

$A_{T R}$ is a complex amplitude function that includes the transmitter and receiver antenna beam patterns, the transmitted waveforms, geometrical spreading factors, etc. We assume that $A_{T R}$ varies slowly in $\omega$.

We refer to $\mathcal{F}$ in (1) as the forward model for Bi-SAR GMTI.

\section{IMAGE FORMATION}

We reconstruct the surface reflectivity and determine the velocity of a moving scene in two steps. In the first step, we use an FBP-type method to reconstruct the scene reflectivity assuming that the scene is moving at a constant hypothesized velocity. We design a spatially varying filter such that the reflectivity image of a scatterer is well-focused whenever the hypothesized velocity matches the true velocity of the scatterer. In the second step, we use the image entropy to measure the degree to which the reflectivity images are focused and to determine the velocity of scatterers from a set of reflectivity images formed using a range of hypothesized velocities.

\section{A. The Imaging Operator}

We form the reflectivity image using a hypothesized velocity $\boldsymbol{v}_{h}$ as follows:

$$
\begin{aligned}
& \hat{q}_{\boldsymbol{v}_{h}}\left(\boldsymbol{x}^{\prime}\right)=\mathcal{K}_{\boldsymbol{v}_{h}}[d]\left(\boldsymbol{x}^{\prime}\right) \\
& \quad=\int e^{i \phi_{T R}\left(\omega, s, t, \boldsymbol{x}^{\prime}, \boldsymbol{v}_{h}\right)} Q_{\boldsymbol{v}_{h}}\left(\omega, s, \boldsymbol{x}^{\prime}\right) d(s, t) d t d \omega d s,
\end{aligned}
$$

where $\hat{q}_{\boldsymbol{v}_{h}}\left(\boldsymbol{x}^{\prime}\right)$ is the reconstructed reflectivity image using the hypothesized velocity $\boldsymbol{v}_{h}$ and $Q_{\boldsymbol{v}_{h}}\left(\omega, s, \boldsymbol{x}^{\prime}\right)$ is the filter to be determined. Like before, we assume that $Q_{\boldsymbol{v}_{h}}$ is a slowly varying function of $\omega$.

Substituting $d(s, t)$ given in (1) into (5) results in

$$
\hat{q}_{\boldsymbol{v}_{h}}\left(\boldsymbol{x}^{\prime}\right)=\int L_{\boldsymbol{v}_{h}}^{\boldsymbol{v}_{\mathbf{x}}}\left(\boldsymbol{x}^{\prime}, \boldsymbol{x}\right) q\left(\boldsymbol{x}, \boldsymbol{v}_{\mathbf{x}}\right) d \boldsymbol{x},
$$

where $L_{\boldsymbol{v}_{h}}^{\boldsymbol{v}_{\boldsymbol{x}}}\left(\boldsymbol{x}^{\prime}, \boldsymbol{x}\right)$ is the point spread function (PSF) of $\mathcal{K}_{\boldsymbol{v}_{h}}$ and given as:

$$
\begin{aligned}
L_{\boldsymbol{v}_{h}}^{\boldsymbol{v}_{\mathbf{x}}}\left(\boldsymbol{x}^{\prime}, \boldsymbol{x}\right)= & \int e^{\mathrm{i} \Phi_{\boldsymbol{v}_{h}}\left(\omega, s, \boldsymbol{x}^{\prime}, \boldsymbol{x}, \boldsymbol{v}_{\mathbf{x}}\right)} \\
& \times A_{T R}\left(\omega, s, \boldsymbol{x}, \boldsymbol{v}_{\mathbf{x}}\right) Q_{\boldsymbol{v}_{h}}\left(\omega, s, \boldsymbol{x}^{\prime}\right) d \omega d s,
\end{aligned}
$$

where

$$
\begin{array}{r}
\Phi_{\boldsymbol{v}_{h}}\left(\omega, s, \boldsymbol{x}^{\prime}, \boldsymbol{x}, \boldsymbol{v}_{\mathbf{x}}\right)=\omega / c_{0}\left[R_{T R}(s, \boldsymbol{x})+B_{T R}\left(s, \boldsymbol{x}, \boldsymbol{v}_{\mathbf{x}}\right)\right. \\
\left.-R_{T R}\left(s, \boldsymbol{x}^{\prime}\right)-B_{T R}\left(s, \boldsymbol{x}^{\prime}, \boldsymbol{v}_{h}\right)\right] .
\end{array}
$$

The major contributions to the PSF come from the critical points of the phase $\Phi_{\boldsymbol{v}_{h}}$ given by

$\partial \omega\left[\Phi_{\boldsymbol{v}_{h}}\left(\omega, s, \boldsymbol{x}^{\prime}, \boldsymbol{x}, \boldsymbol{v}_{\mathbf{x}}\right)\right]=0 \Rightarrow$

$R_{T R}(s, \boldsymbol{x})+B_{T R}\left(s, \boldsymbol{x}, \boldsymbol{v}_{\mathbf{x}}\right)=R_{T R}\left(s, \boldsymbol{x}^{\prime}\right)+B_{T R}\left(s, \boldsymbol{x}^{\prime}, \boldsymbol{v}_{h}\right)$,

$\partial s\left[\Phi_{\boldsymbol{v}_{h}}\left(\omega, s, \boldsymbol{x}^{\prime}, \boldsymbol{x}, \boldsymbol{v}_{\mathbf{x}}\right)\right]=0 \Rightarrow$

$\dot{R}_{T R}(s, \boldsymbol{x})+\dot{B}_{T R}\left(s, \boldsymbol{x}, \boldsymbol{v}_{\mathbf{x}}\right)=\dot{R}_{T R}\left(s, \boldsymbol{x}^{\prime}\right)+\dot{B}_{T R}\left(s, \boldsymbol{x}^{\prime}, \boldsymbol{v}_{h}\right)$,

where $\dot{R}_{T R}=\partial s R_{T R}$ and $\dot{B}_{T R}=\partial s B_{T R}$. For a fixed $\boldsymbol{x}^{\prime}$ and fixed $\boldsymbol{v}_{h}=\boldsymbol{v}_{\mathbf{x}}$, (9) and (10) represent the iso-range and iso-Doppler contours, respectively. The critical points of the phase of $\mathcal{K}_{\boldsymbol{v}_{h}} \mathcal{F}$ that contribute to the reconstructed reflectivity images lie at the intersections of these contours shown in Fig. 2. When the hypothesized velocity is correct, i.e. $\boldsymbol{v}_{h}=\boldsymbol{v}_{\mathbf{x}}$, these intersections show the location of the reconstructed targets. In contrast, when $\boldsymbol{v}_{h}$ differs from $\boldsymbol{v}_{\mathbf{x}}$, the reconstructed reflectivity image may contain artifacts due to incorrect positioning of the targets.

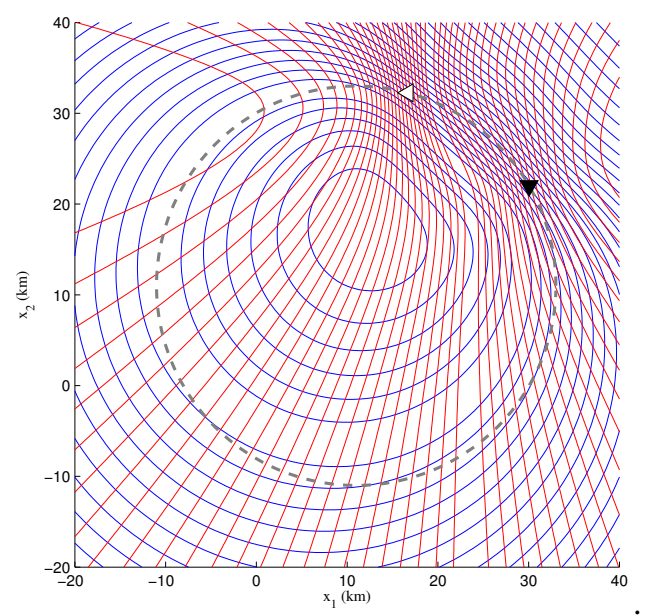

Fig. 2. Position-space iso-range (blue lines) and iso-Doppler (red lines) contours for a circular flight trajectory $\gamma_{R}(s)=\gamma_{T}(s+\pi / 4)$ over a flat topography. The target moves with velocity $[30,30] \mathrm{km} / \mathrm{hr}$. Black and white triangles denote the transmitter and receiver locations, respectively.

For the rest of the discussion, we assume that antenna beam patterns and flight trajectories are chosen such that iso-range and iso-Doppler contours intersect at a single point. Thus, we 
assume that the only critical point of intersection is $\boldsymbol{x}^{\prime}=\boldsymbol{x}$, when $\boldsymbol{v}_{h}=\boldsymbol{v}_{\mathbf{x}}$.

We wish to design a filter at $\boldsymbol{x}$ so that the PSF at $\boldsymbol{x}^{\prime}$ approximates a Dirac delta function when $\boldsymbol{v}_{h}=\boldsymbol{v}_{\mathbf{x}}$, i.e.,

$$
L_{\boldsymbol{v}_{\mathbf{x}}}^{\boldsymbol{v}_{\mathbf{x}}}\left(\boldsymbol{x}^{\prime}, \boldsymbol{x}\right) \approx \delta\left(\boldsymbol{x}-\boldsymbol{x}^{\prime}\right)=\int e^{i\left(\boldsymbol{x}-\boldsymbol{x}^{\prime}\right) \cdot \boldsymbol{\xi}} d \boldsymbol{\xi} .
$$

We make the Taylor series approximation around $\boldsymbol{x}=\boldsymbol{x}^{\prime}$ in the phase $\Phi_{\boldsymbol{v}_{h}}\left(\omega, s, \boldsymbol{x}^{\prime}, \boldsymbol{x}, \boldsymbol{v}_{\mathbf{x}}\right)$ of the PSF and write

$$
\Phi_{\boldsymbol{v}_{\mathbf{x}}}\left(\omega, s, \boldsymbol{x}^{\prime}, \boldsymbol{x}, \boldsymbol{v}_{\mathbf{x}}\right) \approx\left(\boldsymbol{x}-\boldsymbol{x}^{\prime}\right) \cdot \frac{\omega}{c_{0}} \boldsymbol{\Xi}_{\boldsymbol{v}_{\mathbf{x}}}\left(s, \boldsymbol{x}^{\prime}\right),
$$

where $\boldsymbol{\Xi}_{\boldsymbol{v}_{\mathbf{x}}}\left(s, \boldsymbol{x}^{\prime}\right)=\left.\nabla_{\boldsymbol{x}}\left[R_{T R}(s, \boldsymbol{x})+B_{T R}\left(s, \boldsymbol{x}, \boldsymbol{v}_{\mathbf{x}}\right)\right]\right|_{\boldsymbol{x}=\boldsymbol{x}^{\prime}}$.

For a fixed hypothesized velocity $\boldsymbol{v}_{h}$ and $\boldsymbol{x}^{\prime}$, we make the change of variables:

$$
(\omega, s) \rightarrow \boldsymbol{\xi}=\frac{\omega}{c_{0}} \boldsymbol{\Xi}_{\boldsymbol{v}_{h}}\left(s, \boldsymbol{x}^{\prime}\right) .
$$

Then, $L_{\boldsymbol{v}_{h}}^{\boldsymbol{v}_{\mathbf{x}}}\left(\boldsymbol{x}^{\prime}, \boldsymbol{x}\right)$ in (7) can be approximated as:

$$
\begin{aligned}
L_{\boldsymbol{v}_{h}}^{\boldsymbol{v}_{\mathbf{x}}}\left(\boldsymbol{x}^{\prime}, \boldsymbol{x}\right) & \approx \int e^{i\left(\boldsymbol{x}-\boldsymbol{x}^{\prime}\right) \cdot \boldsymbol{\xi}} \\
& \times A_{T R}\left(\boldsymbol{\xi}, \boldsymbol{x}, \boldsymbol{v}_{\mathbf{x}}\right) Q_{\boldsymbol{v}_{h}}\left(\boldsymbol{\xi}, \boldsymbol{x}^{\prime}\right) \eta_{\boldsymbol{v}_{h}}\left(\boldsymbol{\xi}, \boldsymbol{x}^{\prime}\right) d \boldsymbol{\xi},
\end{aligned}
$$

where $\eta_{\boldsymbol{v}_{h}}$ is the determinant of the Jacobian that comes from the change of variables in (13) and is given as

$$
\eta_{\boldsymbol{v}_{h}}\left(\boldsymbol{\xi}, \boldsymbol{x}^{\prime}\right)=\left|\frac{\partial(\omega, s)}{\partial \boldsymbol{\xi}}\right| \text {. }
$$

Then, the desired filter $Q_{T R}$ becomes

$$
Q_{T R}\left(\boldsymbol{\xi}, \boldsymbol{x}^{\prime}, \boldsymbol{v}_{h}\right)=\frac{\overline{A_{T R}\left(\boldsymbol{\xi}, \boldsymbol{x}^{\prime}, \boldsymbol{v}_{h}\right)}}{\left|A_{T R}\left(\boldsymbol{\xi}, \boldsymbol{x}^{\prime}, \boldsymbol{v}_{h}\right)\right|^{2}} \frac{\chi_{\Omega_{\boldsymbol{x}^{\prime}, \boldsymbol{v}_{h}}}}{\eta_{\boldsymbol{v}_{h}}\left(\boldsymbol{\xi}, \boldsymbol{x}^{\prime}\right)},
$$

where $\chi_{\Omega_{\boldsymbol{x}^{\prime}, \boldsymbol{v}_{h}}}$ is a smooth cut-off function that prevents division by zero.

\section{B. Velocity Estimation Method}

Our FBP image reconstruction method produces a set of reflectivity images, each one corresponding to a different hypothesized velocity. A moving target is reconstructed at the correct position whenever the hypothesized velocity is equal to the true velocity of the target. When the hypothesized velocity deviates from the true velocity, the reconstructed reflectivity image contains smearing artifacts due to incorrect positioning of the target.

We measure the degree to which the image is focused by the Renyi entropy metric defined as [11], [12]

$$
\varepsilon\left(\boldsymbol{v}_{h}\right)=-\log \sum_{x^{\prime}} p\left(\hat{q}_{\boldsymbol{v}_{h}}\left(x^{\prime}\right)\right)^{2},
$$

where $p$ is a normalized histogram for the image. Entropy is a widely used metric to quantify the smoothness of images [11], [12].

We calculate the entropy $\varepsilon\left(\boldsymbol{v}_{h}\right)$ of each of the reflectivity images and choose the velocities corresponding to the local minima of $\varepsilon\left(\boldsymbol{v}_{h}\right)$ as the velocity estimates of moving targets.

\section{NumERicAl Simulations}

We perform numerical experiments using a Bi-SAR geometry where the transmitter and receiver traverse a circular trajectory in tandem. We consider a scene of size $4096 \times$ $4096 \mathrm{~m}^{2}$ with flat topography centered at $[11,11,0] \mathrm{km}$. The scene is discretized into $128 \times 128$ pixels, where $[0,0,0] \mathrm{m}$ and $[4096,4096,0] \mathrm{m}$ correspond to the pixels $(1,1)$ and $(128$, 128), respectively. We assume that a point moving target with unit reflectivity is located at the upper left quadrant of the scene at time $s=0$ moving with a velocity $\boldsymbol{v}_{\mathbf{x}}=[40,-30]$ $\mathrm{km} / \mathrm{hr}$, so $\left|\boldsymbol{v}_{\mathbf{x}}\right|=50 \mathrm{~km} / \mathrm{hr}$. We sample the flight trajectory $\gamma(s)=(11+11 \cos (s), 11+11 \sin (s), 6.5) \mathrm{km}, s \in\left[\begin{array}{ll}0 & 2 \pi\end{array}\right]$ uniformly at 512 points. We set the transmitter trajectory to $\gamma_{T}(s)=\gamma(s)$ and the receiver trajectory to $\gamma_{R}(s)=$ $\gamma(s+\pi / 4)$. The speed of the antennas is kept constant at $950 \mathrm{~km} / \mathrm{hr}$ throughout the aperture. Fig. 3 shows the 2-D view of the scene with the target and antenna trajectories. The reflectivity images are reconstructed using a range of

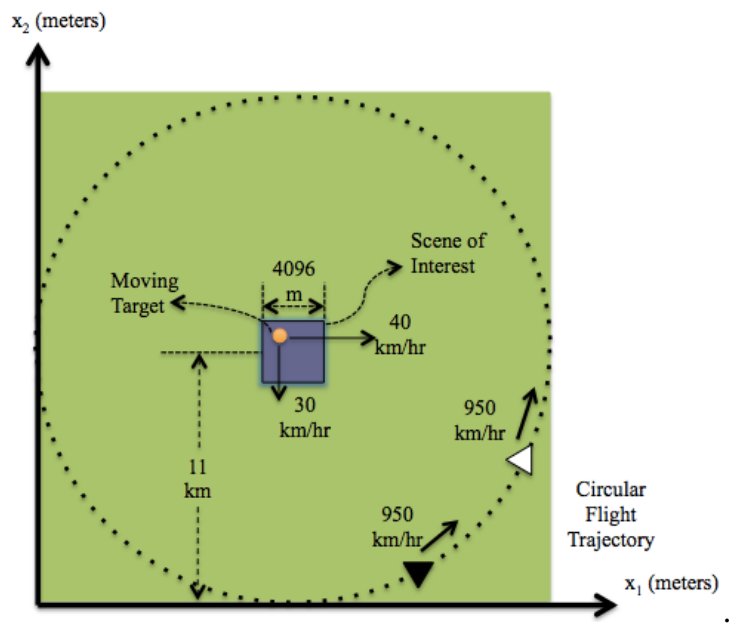

Fig. 3. 2-D illustration of the simulation setup for a single moving target using bi-static antennas. The dark region shows the scene considered. The orange dot shows the position of the target with the arrows indicating its velocity components. The antennas traverse a circular flight trajectory (dashed line), where black and white triangles show the transmitter and receiver locations, respectively.

hypothesized velocities from $30 \mathrm{~km} / \mathrm{hr}$ to $50 \mathrm{~km} / \mathrm{hr}$ and $40 \mathrm{~km} / \mathrm{hr}$ to $-20 \mathrm{~km} / \mathrm{hr}$ at $1 \mathrm{~km} / \mathrm{hr}$ increments for the first and second component of the velocity vector, respectively. The Renyi entropy of each of the backprojected image is calculated and shown in the mesh plot in Fig. 4. This figure shows that the entropy is minimum when $\boldsymbol{v}_{h}=[40,-30] \mathrm{km} / \mathrm{hr}$ which is the estimated velocity of the target. Since the velocity of the target is estimated correctly, the final reconstructed image using this velocity focuses the target at its correct position as shown in Fig. 5. For comparison, Fig. 6 shows the images reconstructed using $\boldsymbol{v}_{h}=\boldsymbol{v}_{\mathbf{x}} / 2$ and $\boldsymbol{v}_{h}=[0,0] \mathrm{km} / \mathrm{hr}$. Clearly, using incorrect velocity vectors produces smearing artifacts in the reconstructed reflectivity images.

\section{CONCLUSION}

In this work, we presented a new method to reconstruct the surface reflectivity and velocity of ground moving targets using bi-static SAR configurations. We presented a forward model that maps the reflectivity and velocity of the targets to the received signal. We used FBP type reconstruction method combined with the Renyi entropy measure to reconstruct the reflectivity images and to determine the velocity field of the scene. Simulation results show that for a single moving target, we estimate the velocity of the target correctly and reconstruct a well-focused reflectivity image. The bi-static SAR moving 


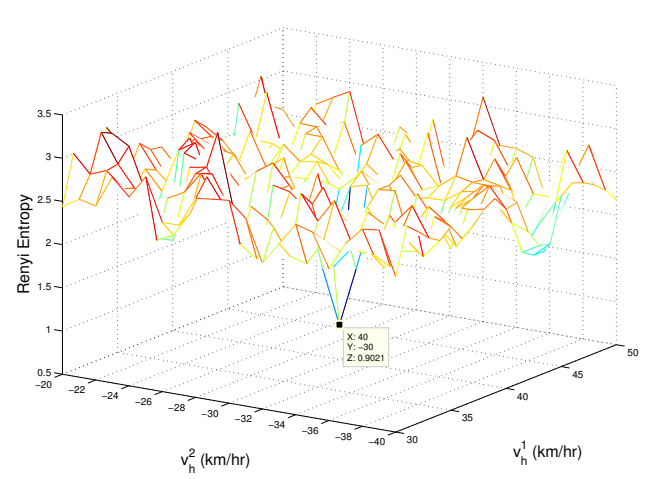

Fig. 4. Renyi entropies of the reconstructed images for different hypothesized velocities $\boldsymbol{v}_{h}=\left[\boldsymbol{v}_{h}^{1}, \boldsymbol{v}_{h}^{2}\right] \mathrm{km} / \mathrm{hr}$. The minimum entropy is indicated at $\boldsymbol{v}_{h}=$ $[40,-30] \mathrm{km} / \mathrm{hr}$.

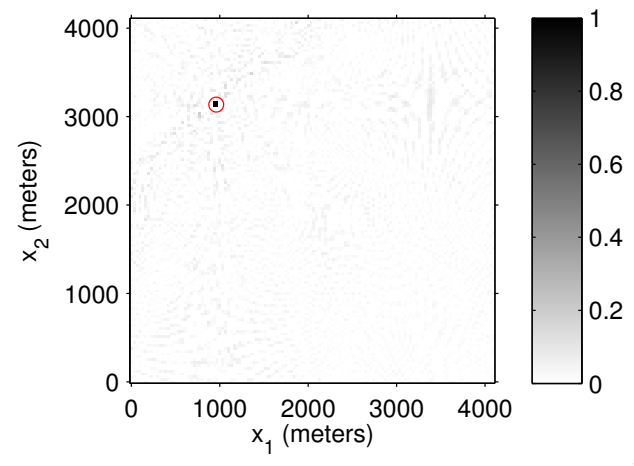

Fig. 5. The reflectivity image reconstructed using the estimated velocity [ 40 , -30] $\mathrm{km} / \mathrm{hr}$. Since the estimated velocity matches the true velocity, the target is well-focused in the image reconstructed.(Empty circle indicates the initial position of the target.)

target imaging method that we presented in this paper can be also extended to other SAR modalities, such as [13]-[17].

\section{ACKNOWLEDGMENT}

This work was supported by the Air Force Office of Scientific Research (AFOSR) under the agreements FA9550-091-0013 and FA9550-12-1-0415, and by the National Science Foundation (NSF) under Grant No. CCF-08030672 and CCF1218805 .

\section{REFERENCES}

[1] C. E. Yarman, B. Yazıc1, and M. Cheney, "Bistatic synthetic aperture radar imaging for arbitrary flight trajectories," IEEE Trans. on Image Proc., vol. 17, no. 1, pp. 84-93, Jan. 2008.

[2] N.J. Willis, Bistatic radar, SciTech Publishing, 2005.

[3] W.W. Goj, Synthetic-aperture radar and electronic warfare, Artech House, 1993.

[4] M.J. Minardi, L.A. Gorham, and E.G. Zelnio, "Ground moving target detection and tracking based on generalized sar processing and change detection," in Proc. of the SPIE, 2005, vol. 5808.

[5] I. Bradaric, G.T. Capraro, D. D. Weiner, and M. C. Wicks, "Multistatic radar systems signal processing," in IEEE Conf. on Radar. IEEE, 2006.

[6] V.T. Vu, T.K. Sjogren, and M.I. Pettersson, "Space time adaptive processing for moving target detection and imaging in bistatic sar," in Geoscience and Remote Sensing Symposium (IGARSS). IEEE, 2011, pp. 2829-2832.

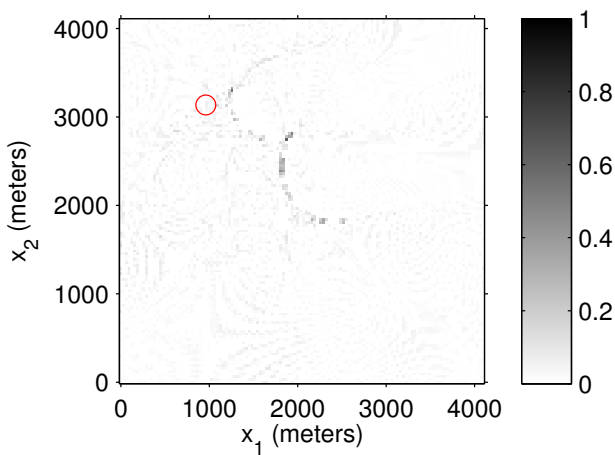

(a) $\left.\hat{q}_{\boldsymbol{v}_{h}}\left(\boldsymbol{x}^{\prime}\right)\right|_{\boldsymbol{v}_{h}=\boldsymbol{v}_{\mathbf{x}} / 2 \mathrm{~km} / \mathrm{hr}}$

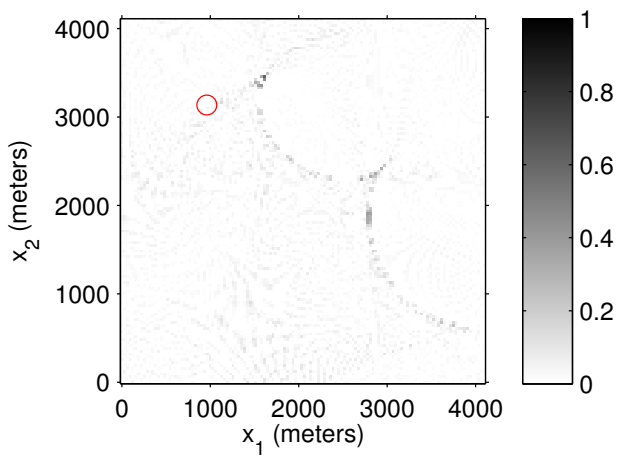

(b) $\left.\hat{q}_{\boldsymbol{v}_{h}}\left(\boldsymbol{x}^{\prime}\right)\right|_{\boldsymbol{v}_{h}=0 \mathrm{~km} / \mathrm{hr}}$

Fig. 6. Reflectivity images reconstructed using (a) $\boldsymbol{v}_{h}=\boldsymbol{v}_{\mathrm{x}} / 2 \mathrm{~km} / \mathrm{hr}$ and (b) $\boldsymbol{v}_{h}=[0,0] \mathrm{km} / \mathrm{hr}$. The target appears more smeared as we diverge from the true velocity.(Empty circle indicates the initial position of the target.)

[7] D. Vu, B. Guo, L. Zhou, and J. Li, "Ground moving target indication via multi-channel airborne SAR," in Proc. of the SPIE, 2011, vol. 8051, pp. Q1-Q12.

[8] M. Soumekh, "Moving target detection in foliage using along track monopulse synthetic aperture radar imaging," IEEE Trans. on Image Proc., vol. 6, no. 8, pp. 1148-1163, 1997.

[9] L. Wang and B. Yazici, "Passive imaging of moving targets exploiting multiple scattering using sparse distributed apertures," Inverse Problems, vol. 28, no. 12, 2012.

[10] L. Demanet, M. Ferrara, N. Maxwell, J. Poulson, and L. Ying, "A butterfly algorithm for synthetic aperture radar imaging," Dimensionality Reduction of Large Scale Forward and Inverse EM Problems, 2010.

[11] T.J. Kragh, "Minimum-entropy autofocus for three-dimensional sar imaging," in Proc. of the SPIE, 2009, vol. 7337.

[12] N. Boss, E. Ertin, and R. Moses, "Autofocus for 3d imaging with multipass sar," in Proc. of the SPIE, 2010

[13] L. Wang and B. Yazıcı, "Bistatic synthetic aperture radar imaging using ultranarrowband continuous waveforms," IEEE Trans. on Image Proc., vol. 21, no. 8, 2012.

[14] L. Wang, C. E. Yarman, and B. Yazıcı, "Doppler-hitchhiker: A novel passive synthetic aperture radar using ultranarrowband sources of opportunity," IEEE Trans. on Geoscience and Remote Sensing, vol. 49, no. 10, pp. 3521-3537, 2011.

[15] V. Krishnan and B. Yazıc1, "Synthetic aperture radar imaging exploiting multiple scattering," Inverse Problems, vol. 27, no. 5, 2011.

[16] C. E. Yarman, L. Wang, and B. Yazıc1, "Doppler synthetic aperture hitchhiker imaging," Inverse Problems, vol. 26, no. 6, 2010.

[17] V. Krishnan, J. Swoboda, C.E. Yarman, and B. Yazıc1, "Multistatic synthetic aperture radar image formation," IEEE Trans. on Image Proc., vol. 19, no. 5, pp. 1290-1306, 2010 